\title{
Relevance of SIRS and Sepsis in Pediatric Liver Transplantation
}

\author{
M. Sasse1, M. Boehne1, I. Forstmeyer'1, N. Richter ${ }^{2}$, F. Lehner ${ }^{3}$, \\ H. Köditz1 ${ }^{1}$ T. Kaussen1, K. Seidemann ${ }^{*} \#$, T. Jack ${ }^{1 \#}$
}

${ }^{1}$ Department of Pediatric Cardiology and Intensive Care Medicine, Hannover Medical School, Hannover, Germany ${ }^{2}$ Department of General, Visceral and Transplantation Surgery, Hannover Medical School, Hannover, Germany ${ }^{3}$ Department of General and Visceral Surgery, Helios Klinikum Hildesheim, Germany

Email: *Seidemann.Kathrin@mh-hannover.de

How to cite this paper: Sasse, M., Boehne, M., Forstmeyer, I., Richter, N., Lehner, F., Köditz, H., Kaussen, T., Seidemann, K. and Jack, T. (2021) Relevance of SIRS and Sepsis in Pediatric Liver Transplantation. Journal of Biosciences and Medicines, 9, 131-145. https://doi.org/10.4236/jbm.2021.91011

Received: December 19, 2020

Accepted: January 26, 2021

Published: January 29, 2021

\begin{abstract}
Introduction: Liver transplantation (LT) in children has undergone significant changes over the last years. Especially the use of split and living donor transplants even for infants has led to new challenges in pre- and post-operative care. Systemic inflammatory response syndromes (SIRS, sepsis) as well-known complications after LT have not yet been systematically examined in the pediatric population. Methods: We analyzed clinical data of 39 pediatric liver transplant recipients regarding potential risk factors for post-transplant SIRS and sepsis. Secondly, the prognostic impact of SIRS and sepsis on post-transplant clinical course, patient and transplant-survival has been analyzed. Results: $64 \%$ of patients developed either SIRS $(n=16,41 \%)$ or sepsis $(n=9,23 \%)$ within 30 days after transplantation. No pre-transplant risk factors for increased susceptibility for SIRS or sepsis could be identified. Secondary closure of the abdomen ( $\mathrm{p}=$ $0.045)$ and secondary biliary reconstruction $(p=0.043)$ were associated with a higher incidence of sepsis and were associated with significantly prolonged mechanical ventilation times in the presence of sepsis $(\mathrm{p}=0.001)$. Patients with sepsis, but not SIRS, stayed significantly longer on PICU ( $p=0.021)$ and suffered from higher mortality $(\mathrm{n}=3$ versus $0 ; \mathrm{p}=0.0006)$. All deaths within 30 days of transplantation were due to septic multiorgan failure. Neither early SIRS nor sepsis were associated with loss of transplant function. Conclusions: SIRS and sepsis are frequent events after pediatric liver transplantation. Sepsis increased length of PICU-stay and mortality significantly and prolonged duration of mechanical ventilation. Secondary biliary reconstruction and closure of the abdomen could be identified as potential risk factors for sepsis.
\end{abstract}

\section{Keywords}

Liver Transplantation, SIRS, Sepsis, Risk Factors, Pediatric

${ }^{\#}$ Authors have contributed equally to the research work as well as to the manuscript. 


\section{Introduction}

Liver transplantation (LT) has become an increasingly available therapeutic option for children with acute or chronic end-stage liver failure [1] [2] [3] [4]. More elaborate operative techniques such as split liver transplantation and introduction of living donor LT have contributed to a higher availability of organs even in small children below $10 \mathrm{~kg}$ of weight. For patients with metabolic disorders, like urea cycle defects, LT has also become a realistic therapeutic option [5] [6]. Growing experience in pre-, intra- and postoperative management as well as new developments in immuno-suppressive therapy have contributed to improvements in survival and long-term organ function [1] [2] [4]-[10].

Systemic inflammatory response syndrome (SIRS) and sepsis are characterized by a severe and possibly life-threatening generalized immune response [11] [12]. SIRS, similar to sepsis, might progress to circulatory failure, acute respiratory distress syndrome (ARDS), multiorgan failure and death [13] [14] [15] [16] [17]. Most frequent causes of SIRS in childhood are burns, trauma, polytransfusion, and major surgery, especially abdominal surgery and any kind of surgery requiring extracorporal circulation [17] [18] [19]. Liver transplantation exposes the patient to a high risk for SIRS and sepsis because of abdominal surgery in the presence of significant comorbidities and organ dysfunction, anhepatic phase during transplantation and ischemia/reperfusion injury of liver and gut [20] [21] [22] [23]. Compared to adults, pediatric liver transplant recipients might be at an even higher risk due to more frequently used split liver transplants, previous abdominal surgery, and higher prevalence of repeated or chronic intrahepatic bile duct infections [19] [22]. No studies exist regarding the incidence of SIRS and sepsis after pediatric liver transplantation, the same applies to their potential prognostic impact on post-transplant outcome.

In this retrospective study, we evaluated pediatric liver transplant recipients for SIRS and sepsis after transplantation. The aim of this study was to identify risk factors for SIRS/sepsis and to evaluate their impact on post-transplant mortality, short and long term organ dysfunction, and transplant survival.

\section{Patients and Methods}

\subsection{Study Design}

Retrospective analysis of the clinical course of pediatric liver transplant recipients, admitted to a tertiary level pediatric intensive care unit (PICU, Pediatric Intensive Care Unit, of Medical School Hannover, Germany) between 2005 and 2008, was performed in order to examine incidence and risk factors for SIRS/sepsis and their impact on patient and transplant outcome. The study protocol conformed to the ethical guidelines of the 1975 Declaration of Helsinki.

\subsection{Patient Enrollment and Data Collection}

During the study period, 98 pediatric liver transplant recipients were initially assessed for eligibility. Patients included into other interventional studies in- 
fluencing the perioperative management (e.g. immunosuppressive therapy, use of in-line filtration of drugs and infusions) were excluded from our risk factor analysis. Thus, 39 patients could be included. All patients were treated by an interdisciplinary team of pediatric hepato-gastroenterologists, pediatric intensivists and abdominal transplant surgeons. Pre-, intra- and postoperative care followed a standardized transplantation protocol.

Clinical data such as age, sex, weight, length, diagnoses, disease characteristics, previous operations and pre-operative laboratory data were collected from all enrolled patients on the day of admission to PICU. Data regarding transplant characteristics (living donor versus cadaveric transplantation, split versus whole organ transplantation) were obtained from the operative records as were data regarding intra-operative and post-operative course (transplant ischemia time, duration of anhepatic phase, total duration of transplantation, intraoperative transfusions, etc.). Post-operative clinical and laboratory data regarding organ function and inflammatory response as well as data on long-term follow-up at six, nine, $12-14$ and 18 - 24 months after transplantation were collected prospectively using a standardized protocol.

\subsection{Primary Endpoints}

Occurrence of sepsis and/or systemic inflammatory response syndrome (SIRS) after liver transplantation was defined as the primary endpoint of the study. All episodes of SIRS/sepsis, occurring during the first 30 days after post-transplantation admission to the PICU or until discharge from PICU had been recorded.

SIRS and sepsis were defined according to the International Pediatric Sepsis Consensus Conference (IPSCC) 2005 [12]. SIRS was defined as the presence of at least two of the following four criteria, one of which must be an abnormal temperature or leukocyte count: Core temperature of $>38.5^{\circ} \mathrm{C}$ or $<36^{\circ} \mathrm{C}$; tachycardia defined as mean heart rate $>2 \mathrm{SD}$ above normal for age, mean respiratory rate $>2 \mathrm{SD}$ above normal for age, leukocyte count elevated or depressed for age or $>10 \%$ immature neutrophils. Sepsis was defined as SIRS in the presence of proven or confirmed infection. Patients with preexisting low leukocyte counts secondary to hypersplenism were positively diagnosed for sepsis or SIRS only if at least two other criteria, required for the diagnosis of sepsis or SIRS, were fulfilled. The same applied for patients having received intraoperative polytransfusion (>40 ml/kg packed red blood cells (PRBC) of body weight) with subsequently unreliable leukocyte counts; in these patients, also at least two further criteria for SIRS/sepsis had to be present.

Severe bleeding was defined as the need of more than $40 \mathrm{ml} \mathrm{PRBC} / \mathrm{kg}$ of body weight during intraoperative course. Graft to recipient size mismatch was defined according to graft weight in relation to body weight of the recipient (graft-recipient ratio). Graft weight of less than $2 \%$ of patient body weight was defined as "small for size", graft size of more than $4 \%$ of patient body weight was defined as "large for size" [24] [25]. 


\subsection{Secondary Endpoints}

In addition to the analysis of the incidence of SIRS and sepsis after liver transplantation, we evaluated pre- and intraoperative risk factors for the development of SIRS and/or sepsis. The following potential preoperative risk factors were analyzed regarding their impact on organ dysfunction and occurrence of SIRS/sepsis: previous abdominal surgery, body weight less than $10 \mathrm{~kg}$, acute liver failure, hospital stay prior to transplantation, and PICU stay prior to transplantation. In addition, the following intraoperative risk factors were analyzed: living versus cadaveric transplantation, secondary biliary reconstruction, secondary closure of the abdomen, cold ischemia time $(>600 \mathrm{~min})$, intraoperative polytransfusion ( $>40 \mathrm{ml} / \mathrm{kg}$ body weight (BW) of PRBC) and duration of operation (>300 min) (see Table 3, Table 4). All parameters were analyzed regarding their potential impact on the development of inflammatory syndromes and organ dysfunction (circulatory, renal, lung, hepatic, neurological and hematological dysfunction according to IPSCC [9]) in the postoperative course.

All episodes of SIRS and sepsis during total hospital stay until discharge or death were evaluated regarding their impact on patient and transplant outcome. However, occurrence of SIRS more than four weeks after transplantation is likely not to be related to pre- and perioperative risk factors for SIRS/sepsis. The risk factor analysis of pre- and perioperative clinical features for the development of SIRS and/or sepsis therefore only considered clinical data and SIRS/sepsis episodes that occurred within the first 30 days after transplantation or retransplantation.

Patients were defined as dischargeable from the PICU when they were no longer in need for any vital organ support according to the standardized transplant protocol. Duration of mechanical ventilation was assessed in hours; extubation criteria were defined according to a standardized protocol for the assessment of extubation readiness.

To evaluate the potential impact of inflammatory syndromes on long term outcome, we analyzed patient data at six, nine, $12-14$ and 18 - 24 months after transplantation with regard to transplant survival and function, judged by the measurement of aPTT, INR and bilirubin levels. Additionally, all severe events and reasons for retransplantation were recorded independent of the time of occurrence in relation to transplantation (e.g. thrombosis of transplant artery or portal vein).

\subsection{Statistics}

Statistical analysis was performed with the use of Predictive Analysis Software for Windows (PASW), version 18. Statistical analysis included univariate analysis using Chi-square- or Fisher-Exact-test when indicated to identify risk factors for SIRS/sepsis. In case that several clinical features exhibited statistical significance in univariate analysis, multivariate analysis was to be performed in order to eliminate confounding variables. For the analysis of SIRS/sepsis as prognostic factor after liver transplantation, patients were divided into two groups (SIRS/sepsis 
versus no evidence of SIRS/sepsis), differences between both groups were described using Pearson's Chi-square or Fisher Exact-test when indicated, and log-rank test. A difference was supposed to be statistically significant, if the estimated probability of error was below $5 \%$.

\section{Results}

\subsection{Patient Characteristics}

39 pediatric liver transplant recipients were enrolled and retrospectively analyzed. 19 patients were male (49\%), 20 were female (51\%). Median age was 7.8 years (range 28 days to 17 years), median weight $19.5 \mathrm{~kg}$ (3.7 to $72 \mathrm{~kg}$ ). Eleven patients (28\%) weighed less than $10 \mathrm{~kg}$. 30 of 39 patients (77\%) had undergone previous abdominal surgery prior to liver transplantation. For detailed description of preoperative clinical characteristics and transplant data see Table 1 \& Table 2. 34 of 39 patients received a cadaver transplant, five patients (10.3\%) received a split living donor transplant. Of the 34 cadaver transplants, 14 were full-size organs and 20 were split transplants. Two patients received a combined liver and kidney transplantation. Median operation time was $280 \mathrm{~min}$ (range 145 - $438 \mathrm{~min}$ ), median cold ischemia time was $590 \mathrm{~min}$ (range 145 - $840 \mathrm{~min}$ ). Median intraoperative red blood cell transfusion requirements were $54 \mathrm{ml} / \mathrm{kg}$ BW (range 0 - $458 \mathrm{ml} / \mathrm{kg} \mathrm{BW}$ ).

Main complications requiring unplanned operative revision were: severe bleeding $(\mathrm{n}=6,15 \%)$, and occlusion of either hepatic artery $(\mathrm{n}=6,15 \%)$ or portal vein $(\mathrm{n}=4,10 \%)$.

Ten patients $(25.6 \%)$ required retransplantation, eight of them during their stay on PICU (median after 13 days (range 5 - 67 days)). In all of these patients,

Table 1. Clinical characteristics and diagnoses of liver transplant recipients.

\begin{tabular}{ccc}
\hline Parameter & Median & Range \\
\hline Age (months) & 98 & $1-204$ \\
Body weight $(\mathrm{kg})$ & 19.5 & $3.7-72$ \\
\hline Underlying disease & Number (n) & Percent \\
\hline Biliary atresia & 20 & $51.3 \%$ \\
Alagille syndrome & 5 & $12.8 \%$ \\
Polycystic kidney/liver disease & 4 & $10.2 \%$ \\
Wilson's disease & 3 & $7.7 \%$ \\
Progressive intrahepatic cholestasis & 2 & $5.1 \%$ \\
Acute liver failure of unknown origin & 2 & $5.1 \%$ \\
Hepatoblastoma & 1 & $2.6 \%$ \\
Hyperoxaluria type 2 & 1 & $2.6 \%$ \\
Niemann-Pick disease & 1 & $2.6 \%$
\end{tabular}

*Patient was transplanted due to acute liver failure prior to availability of diagnostic results. 
Table 2. Pre- and intraoperative characteristics of 39 included patients.

\begin{tabular}{ccc}
\hline Organ/transplant characteristics & N of 39 & Percent \\
\hline Living donor transplant & 5 & $12.8 \%$ \\
Cadaver transplant & 34 & $87.2 \%$ \\
Full size organ & 14 & $35.9 \%$ \\
Split transplant & 25 & $64.1 \%$ \\
Use of previous Roux-Y-loop & 15 & $38.5 \%$ \\
Secondary biliary reconstruction & 8 & $20.5 \%$ \\
Secondary abdominal closure & 29 & $74.4 \%$ \\
\hline Quantitative operative parameters & Median & Range \\
\hline Cold ischemia time (min) & 590 & $145-840$ \\
Packed red blood cells (ml/kg BW) & 54 & $0-458$ \\
Duration of operation (min) & 280 & $145-438$ \\
\hline
\end{tabular}

vascular events (arterial occlusion $n=5$, portal vein occlusion $n=2$, occlusion of both vessels $n=1$ ) were the reason for early retransplantation. These vascular events were independent of transplant size mismatch: Organ size mismatch (graft to recipient) was neither associated with vascular problems nor with the need for retransplantation.

\subsection{Incidence of Early SIRS and Sepsis}

The majority of patients ( $\mathrm{n}=25$ of $39,64 \%)$ underwent at least one episode of SIRS or sepsis within 30 days after transplantation. 16 of 39 patients (41\%) suffered from SIRS; nine of 39 patients (23\%) suffered from sepsis. All septic patients underwent secondary abdominal closure and developed sepsis while the abdomen was still open; in eight of these patients, secondary anastomosis of the bile ducts was the cause for open abdomen after transplantation.

All patients with sepsis had a positive culture, either from a primary sterile site (7/9), wound smear (1/9) or tracheal secretion (1/9). 7 of 9 patients had a positive culture of enterobacteria. In 4 of these patients, positive culture resulted from intraoperative smear, in one case from a wound smear (skin), in another patient from a bile duct drain and once from an ascites drain. One patient had a catheter related sepsis with Staphylococcus epidermidis, and one patient presented with ventilator associated pneumonia with Staphylococci subspecies.

\subsection{Pre- and Intraoperative Risk Factors Analysis for SIRS/Sepsis and Organ Dysfunctions}

Results of univariate risk factor analysis regarding the association of pre-operative and transplantation-related clinical data with post-operative SIRS and sepsis are shown in Table 3 and Table 4. In summary, neither previous abdominal surgery, nor body weight $<10 \mathrm{~kg}$, nor acute liver failure, nor portal vein thrombosis as cause for chronic liver failure, nor pre-transplant stay on ICU were associated 
Table 3. Risk factor analysis for SIRS and sepsis, preoperative risks.

\begin{tabular}{cccccccc}
\hline & \multicolumn{3}{c}{ SIRS $(\mathrm{n}=16)$} & \multicolumn{3}{c}{ Sepsis $(\mathrm{n}=9)$} \\
$\begin{array}{c}\text { Pre-transplant parameter } \\
\text { present in n of 39 patients }\end{array}$ & pre-op. parameter & present? & pre-op. parameter present? \\
\cline { 2 - 7 } & yes & no & p & yes & no & p \\
\hline Previous abdominal surgery (30/39) & $14 / 30$ & $2 / 9$ & 0.191 & $7 / 30$ & $2 / 9$ & 0.945 \\
Body weight < 10 kg (11/39) & $4 / 11$ & $12 / 28$ & 0.711 & $2 / 11$ & $7 / 28$ & 0.649 \\
Portal vein thrombosis (4/39) & $1 / 4$ & $15 / 35$ & 0.778 & $1 / 4$ & $8 / 35$ & 0.661 \\
Acute liver failure (5/39) & $3 / 5$ & $13 / 34$ & 0.356 & $0 / 5$ & $9 / 34$ & 0.190 \\
PICU-stay before transplant. (11/39) & $4 / 11$ & $12 / 28$ & 0.771 & $4 / 11$ & $5 / 28$ & 0.217 \\
\hline
\end{tabular}

The table demonstrates potential pre-transplant risk factors for SIRS and sepsis. Figures in left column show presence of clinical parameter in number of patients, figures in middle and right column show frequency in patients with SIRS or sepsis and corresponding $\mathrm{p}$ value. Significant $\mathrm{p}$-values are printed in bold.

Table 4. Risk factor analysis for SIRS and sepsis, intraoperative risk factors.

\begin{tabular}{|c|c|c|c|c|c|c|}
\hline \multirow[t]{2}{*}{$\begin{array}{l}\text { Pre-transplant parameter } \\
\text { present in } n \text { of } 39 \text { patients }\end{array}$} & \multicolumn{3}{|c|}{$\begin{array}{c}\text { SIRS }(\mathrm{n}=16) \\
\text { intra-op. parameter } \\
\text { present? }\end{array}$} & \multicolumn{3}{|c|}{$\begin{array}{c}\text { Sepsis }(\mathrm{n}=9) \\
\text { intra-op. parameter } \\
\text { present? }\end{array}$} \\
\hline & yes & no & $\mathrm{p}$ & yes & no & $\mathrm{p}$ \\
\hline $\begin{array}{l}\text { Living donor transplant (5) } \\
\text { versus cadaver ( } 34)\end{array}$ & $1 / 5$ & $15 / 34$ & 0.306 & $0 / 5$ & $9 / 34$ & 0.190 \\
\hline $\begin{array}{c}\text { Split transplant }(25) \\
\text { versus full size organ (14) }\end{array}$ & $11 / 25$ & $5 / 14$ & 0.614 & $7 / 25$ & $2 / 14$ & 0.330 \\
\hline Secondary biliary reconstruction $(8 / 39)$ & $2 / 8$ & $14 / 31$ & 0.301 & $4 / 8$ & $5 / 31$ & 0.043 \\
\hline Secondary abdominal closure $(11 / 39)$ & $11 / 29$ & $5 / 10$ & 0.503 & $9 / 29$ & $0 / 10$ & 0.045 \\
\hline Cold ischemia time $>600 \mathrm{~min}(18 / 39)$ & $7 / 18$ & $9 / 21$ & 0.802 & $6 / 18$ & $3 / 21$ & 0.159 \\
\hline Packed cell volume $>40 \mathrm{ml} / \mathrm{kg}$ BW $(24 / 39)$ & $8 / 24$ & $8 / 15$ & 0.217 & $7 / 24$ & $2 / 15$ & 0.254 \\
\hline Duration of operation $>300 \min (14 / 39)$ & $5 / 14$ & $11 / 25$ & 0.614 & $4 / 14$ & $5 / 25$ & 0.542 \\
\hline
\end{tabular}

Table demonstrates potential inraoperative risk factors for SIRS and sepsis. Figures in left column show presence of clinical parameter in number of patients out of total of 39 patients, figures in middle and right column show frequency in patients with SIRS/sepsis and corresponding p-value. Significant p-values are printed in bold. BW = body weight.

with the development of SIRS or sepsis after transplantation. Similarly, most of the intraoperative parameters listed in Table 4 could be excluded as risk factors for post-transplant SIRS or sepsis.

Timing of biliary anastomosis and abdominal closure were the only perioperative risk factors found to be associated with sepsis after transplantation: None of the patients with primary biliary reconstruction developed sepsis, whereas 4 of 8 patients (50\%) with secondary biliary anastomosis (and, as a consequence, secondary abdominal closure) suffered from sepsis $(\mathrm{p}=0.043)$. Results were similar for secondary abdominal closure: 9 of 29 patients (31\%) with open abdomen and secondary closure suffered from sepsis, whereas none of the patients with primary abdominal closure $(\mathrm{p}=0.045)$ developed sepsis. The close relation of secondary biliary anastomosis and delayed abdominal wall closure explains 
why both parameters proved to be significantly associated with sepsis in univariate analysis.

Patients were also analyzed regarding potential risk factors for organ dysfunction. Secondary closure of the abdomen was the only factor that was associated with post-transplant respiratory dysfunction: 17 of 29 patients (58.6\%) with secondary closure of the abdomen developed respiratory dysfunction as opposed to only 2 of 10 patients $(20 \%)$ with primary closure of the abdomen $(\mathrm{p}=0.035)$. Interestingly, secondary abdominal closure and secondary biliary anastomosis were not associated with small recipient size of less than $10 \mathrm{~kg}$ body weight or organ size mismatch. All other potential perioperative risk factors (listed in $\mathrm{Ta}$ ble 4) revealed not to be associated with post-transplant organ dysfunction.

\subsection{Prognostic Impact of SIRS/Sepsis on Short Term Outcome}

Patients with SIRS/sepsis required significantly longer mechanical ventilation times (Figure 1). Mean mechanical ventilation time in patients without SIRS/sepsis was 112 hours ( 4.7 days; $95 \%$ confidence interval (CI): 85 - $137 \mathrm{~h}$ ) with maximum ventilation time of 10 days and $50 \%$ of patients being extubated by day 3 after transplantation, whereas patients with SIRS or sepsis remained ventilator-dependent for a mean duration of 743 hours (31 days; 95\% confidence interval: 208 - 1152 hours) $(\mathrm{p}<0.001)$. The significant difference in ventilation times was mainly attributed to the patients with sepsis, in whom ventilation time

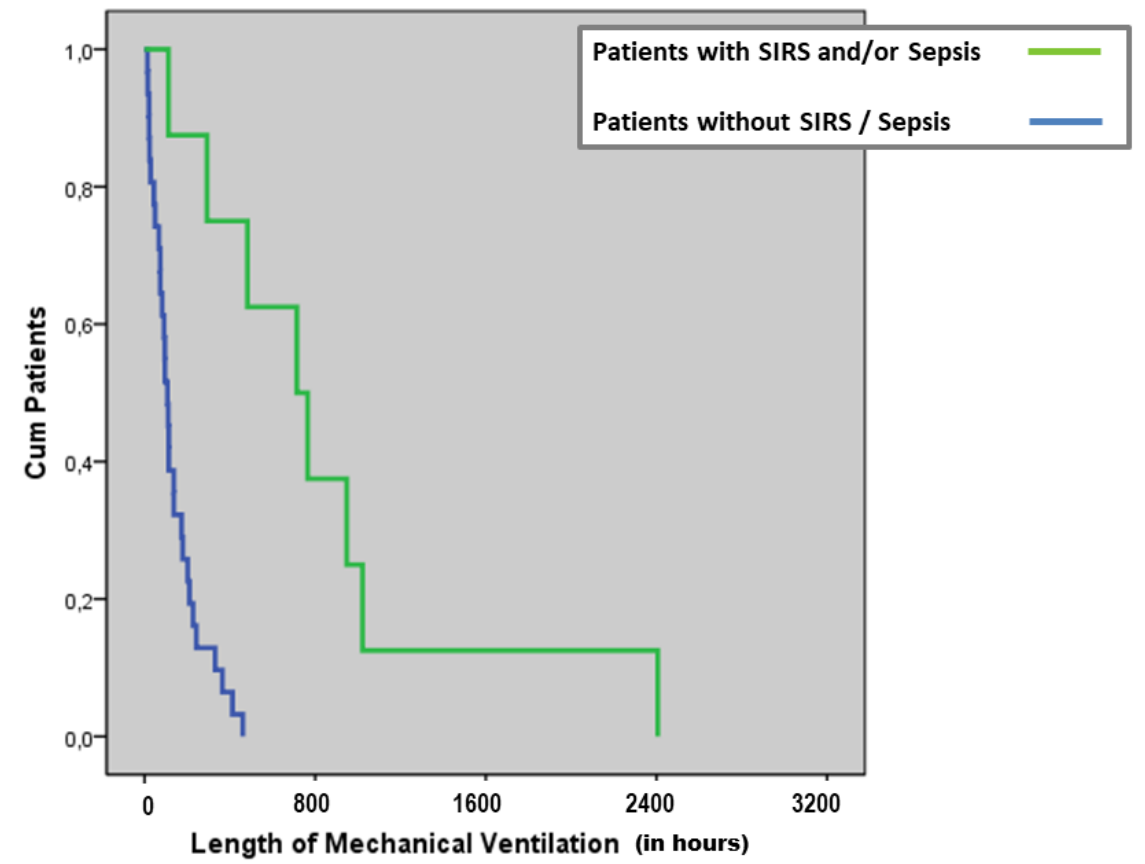

The figure shows log-rank analysis of duration of mechanical ventilation and SIRS/sepsis in 39 pediatric liver transplant recipients. Patients with SIRS and/or sepsis required significantly longer mechanical ventilation after liver transplantation $(\mathrm{p}<0.001)$. Mean mechanical ventilation time in patients without SIRS/Sepsis was 112 hours (95\% confidence interval (CI): 85 - 137 hours) compared to 743 hours (95\% confidence interval: 208 - 1152 hours) in patients with SIRS or sepsis.

Figure 1. Relation between duration of mechanical ventilation and SIRS/sepsis after LTx. 
was 480 hours (95\% CI: 188 - 772 hours) compared to only 112 hours (95\% CI: $81-143 \mathrm{~h} ; \mathrm{p}=0.002)$ in patients without sepsis. In patients with SIRS alone ( $\mathrm{n}=$ 16), duration of ventilation was not significantly prolonged compared to patients without SIRS. Similarly, secondary closure of the abdomen in the absence of sepsis was not associated with prolonged ventilation times.

Despite the significantly longer ventilation time of patients with sepsis, total length of stay in hospital did not differ significantly. However, patients suffering from sepsis stayed significantly longer on the PICU ( $p=0.021 ; 8.3 \mathrm{~d}$ (95\% CI 5.62 - 11.0 d) vs. 21.9 d (95\% CI 16.4 - 27.4 d)).

\subsection{Prognostic Impact of SIRS/Sepsis on Organ Function and Survival}

Long-term transplant function was not associated with the presence of SIRS/sepsis after transplantation: Neither need for retransplantation nor indicators of long-term liver function (i.e. median bilirubin levels, median INR, or median prothrombin time) were associated with the presence of SIRS/sepsis after transplantation. Of ten patients requiring retransplantation, eight were retransplanted early during the primary ICU stay, all of them because of vascular problems. Two patients required late retransplantation after more than six months because of relapsing cholangits $(n=1)$ or refractory cellular rejection $(n=1)$.

Overall mortality after liver transplantation was 13\% (5 of 39), 34 of the 39 transplanted patients (87\%) were alive after 24 months. Three of five deaths occurred within 30 days of transplantation, septic multiorgan failure being the cause of death in all three patients. Two deaths occurred more than six months after transplantation due to secondary malignoma $(n=1)$ or hemophagocytic lymphohistiocytosis $(\mathrm{n}=1)$ in a transplant recipient previously undiagnosed Interleukin-10 receptor defect. None of the patients without sepsis died within the first 30 days after transplantation; death within the first 30 days of transplantation showed to be significantly associated with sepsis $(\mathrm{p}<0.0006)$.

\section{Discussion}

This is the first study examining frequency, risk factors and prognostic impact of SIRS/sepsis after liver transplantation in pediatric patients.

Our study shows that systemic inflammatory syndromes are frequent after liver transplantation, affecting more than half of the patients within 30 days of transplantation. Regarding clinical risk factors for the development of SIRS or sepsis, secondary biliary anastomosis and secondary closure of the abdominal wall were the only clinical features to be significantly associated with post-transplant sepsis. Pediatric patients with sepsis after LT appear to suffer from a significantly higher mortality. Patients with sepsis, but not with SIRS, stayed significantly longer on the PICU. However, total time to discharge from hospital could not be attributed to post-transplant SIRS or sepsis, nor could long term compromised organ function. Respiratory dysfunction and prolonged mechanical ventilation 
were the only clinical organ function parameters associated with sepsis, but not with SIRS alone.

Studies on outcome and complications after pediatric liver transplantation are rare with a focus on patient and transplant survival [1] [2] [3] [7] [10]. Hardly any study on peri-operative risk factors for post-transplant complications has been conducted so far, especially not in children [26] [27] [28] [29]. Patient and transplant survival in our monocenter study show to be comparable to other published results and confirm the generally improving outcome after liver transplantation [1] [2] [3] [7] [10]. Overall survival two years after transplantation was $87 \%$ despite the fact, that our patient population included patients with a high pre-operative morbidity (two patients with combined kidney/liver transplantations and seven patients transplanted for failure of previous liver graft). Despite the high rate of very young patients $(28 \%<1$ year of age, $<10 \mathrm{~kg}$ body weight) and the high number of split transplants $(\mathrm{n}=25)$, mortality and transplant outcome data did not differ much from previously published outcome data.

SIRS and sepsis are frequent complications in pediatric intensive care, observed in $12 \%-68 \%$ of pediatric ICU patients [14] [15] [16] [17]. Main risk factors for the development of SIRS on the ICU are young age, major surgery or trauma, infections, and significant comorbidities [14] [15] [16] [17] [20] [30]. Additional pre-operative clinical features, not assessed in this study, such as nutritional status, changes in the intestinal microbiome, presence of chronic or relapsing cholangitis, etc., are usually present in pediatric LT recipients and might be a potentially important contributing factor for the development of perioperative SIRS/sepsis. The high incidence of SIRS/sepsis in liver transplant recipients compared to other pediatric patients with abdominal surgery might be also attributed to the fact that the liver is an important regulator in cytokine-metabolism with inefficient function prior, during and immediately after transplantation [11] [27]. Anhepatic phase during operation and the initial dysfunction of the transplant graft, as well as the ischemia-reperfusion injury, might further contribute to an altered metabolism and subsequently increased risk for SIRS/sepsis [31] [32]. Despite the fact, that these risk factors for post-transplant SIRS/sepsis were present in all patients of our study population, the frequency of post-transplant SIRS/sepsis was still within the quoted overall occurrence in pediatric ICU patients [16]-[21].

The impact of SIRS/sepsis on outcome has not yet been studied in pediatric liver transplant recipients. The presented data clearly showed a significant association between post-transplant sepsis and mortality: All early deaths after transplantation were caused by multiorgan failure secondary to sepsis. But also in surviving patients, SIRS and sepsis had an important impact on outcome: $\mathrm{Pa}$ tients with SIRS/sepsis required significantly longer ventilation times compared to patients without SIRS or sepsis.

Whereas overall mortality was associated with post-transplant sepsis, trans- 
plant failure was not associated with SIRS or sepsis. All transplant failures were caused by vascular complications. However, many of the patients with vascular problems developed SIRS/sepsis shortly after the diagnosis of vascular compromise. It is very likely that secondary SIRS/sepsis further contributed to vascular complications by activation of the coagulation system; however, it was impossible to further assess this issue in the present study due to the small number of patients [31] [32] [33]. So far, only one study focused on the relation between incidence of postoperative surgical complications and secondary abdominal closure in pediatric liver transplantation; however, this study with a rather small patient cohort could not demonstrate any association between vascular or infectious complications with long term outcome or mortality [34].

Transplant function and long-term transplant survival mainly depend on vascular integrity and good portal as well as arterial perfusion of the transplanted organ [34] [35] [36]. The primary intra- and postoperative focus is therefore optimal transplant perfusion. Especially in small children below $10 \mathrm{~kg}$ of body weight, focusing on hepatic perfusion often requires secondary biliary anastomosis, the abdomen is frequently left open until secondary biliary anastomosis [35]. Another reason for a stepwise approach is based on the fact, that patients with acute liver failure without prior portal hypertension are likely to develop a swelling of the gut shortly after reperfusion with increased risk for abdominal compartment syndrome and insufficiency of the biliary anastomosis [34] [35]. However, secondary biliary anastomosis carries the risk of free drainage of bile into the abdomen, which may contribute to the development of SIRS and sepsis, a fact, that may explain the association of SIRS/sepsis with open abdomen and secondary biliary anastomosis in our series.

Limitations: The total number of 39 patients in this mono-center study is rather small. However, it is very difficult to obtain larger patient numbers due to the rarity of liver transplantation in pediatric patients. Recent challenges regarding donor availability made it even more difficult to generate larger patient numbers for detailed or multivariate outcome analyses. On the other hand, patients in the presented cohort have been transplanted during a relatively short period of time with hardly any changes in transplant or immune-suppressive management, rendering the presented cohort a homogenously treated group of patients. However, these retrospective results require confirmation in a larger group of pediatric liver transplant recipients.

Another limitation of this study is the missing continuous postoperative monitoring of intraabdominal pressure, since increased intraabdominal pressure itself is a risk factor for inflammation, peritonitis and altered hepatic function [37] [38].

\section{Conclusion}

Sepsis is associated with increased mortality after pediatric liver transplantation. The prevention of sepsis and SIRS after liver transplantation could reduce respi- 
ratory dysfunction, ventilator dependence as well as length of stay, and could thereby possibly reduce morbidity and mortality. Further prospective trials are necessary in order to discriminate risk factors for outcome and to improve peri-operative care in pediatric liver transplant recipients on the PICU.

\section{Acknowledgements}

We thank the participants and their guardians who volunteered for the study; the staff of the Department of Paediatric Cardiology and Intensive Care Medicine, Hannover Medical School, Hannover, Germany, for their excellent support. We thank PD Dr. Ludwig Hoy, Institute for Statistics (MHH) for his excellent statistical analysis of the dataset.

\section{Conflicts of Interest}

The authors declare no conflicts of interest regarding the publication of this paper.

\section{References}

[1] Bucuvalas, J. (2009) Long-Term Outcomes in Pediatric Liver Transplantation. Liver Transpl, 15, 6-11. https://doi.org/10.1002/lt.21915

[2] Strauss, A., Grabhorn, E., Sornsakrin, M., Briem-Richter, A., Fischer, L., Nashan, B. and Ganschow, R. (2009) Liver Transplantation for Fulminant Hepatic Failure in Infancy: A Single Center Experience. PediatrTranspl, 13, 838-842. https://doi.org/10.1111/j.1399-3046.2008.01071.x

[3] Kim, J.S., Groteluschen, R., Mueller, T., Ganschow, R., Bicak, T., Wilms, C., et al. (2005) Pediatric Transplantation: the Hamburg Experience. Transplantation, 79, 1206-1209. https://doi.org/10.1097/01.TP.0000160758.13505.D2

[4] Rodeck, B., Melter, M., Kardorff, R., Hoyer, P.F., Ringe, B., Burdelski, M., et al. (1996) Liver Transplantation in Children with Chronic End Stage Liver Disease: Factors Influencing Survival after Transplantation. Transplantation, 62, 1071-1076. https://doi.org/10.1097/00007890-199610270-00008

[5] Li, Q.G., Wan, P., Zhang, J.J., et al. (2015) Liver Transplantation for Biliary Atresia: A Single-Center Study from Mainland China. World J Gastroenterol, 21, 9638-9647. https://doi.org/10.3748/wjg.v21.i32.9638

[6] Yua, L., Rayhillb, S.C., Hsuc, E.K. and Landisa, C.S. (2015) Liver Transplantation for Urea Cycle Disorders: Analysis of the United Network for Organ Sharing Database. Transplantation Proceedings, 47, 2413-2418. https://doi.org/10.1016/j.transproceed.2015.09.020

[7] Farmer, D., Venick, R. and McDiarmid, S. (2007) Predictors of Outcomes after Pediatric Liver Transplantation: An Analysis of More Than 800 Cases Performed at a Single Institution. J Am Coll Surg, 204, 904-914. https://doi.org/10.1016/j.jamcollsurg.2007.01.061

[8] Venick, R.S., Farmer, D.G., McDiarmid, S.V., Duffy, J.P., Gordon, S.A., Yersiz, H., et al. (2010) Predictors of Survival Following Liver Transplantation Infants: A Single Center Analysis of Over 200 Cases. Transplantation, 89, 600-605. https://doi.org/10.1097/TP.0b013e3181c5cdc1

[9] Rhee, C., Narsinh, K. and Venickm, R. (2006) Predictors of Clinical Outcome in 
Children Undergoing Orthoptic Liver Transplantation for Acute and Chronic Liver Disease. Liver Transpl, 12, 1347-1356. https://doi.org/10.1002/lt.20806

[10] Clavien, P.A., Camargo Jr., C.A., Croxford, R., Langer, B., Levy, G.A. and Greig, P.D. (1994) Definition and Classification of Negative Outcomes in Solid Organ Transplantation. Application in Liver Transplantation. Ann Surg, 220, 109-120. https://doi.org/10.1097/00000658-199408000-00002

[11] Robertson, C.M. and Coopersmith, C.M. (2006) The Systemic Inflammatory Response Syndrome. Microbes Infect, 8, 1382-1389. https://doi.org/10.1016/j.micinf.2005.12.016

[12] Goldstein, B., Giroir, B. and Randolph, A. (2005) International Pediatric Sepsis Consensus Conference: Definitions for Sepsis and Organ Dysfunction in Pediatrics. Pediatr Crit Care Med, 6, 2-8. https://doi.org/10.1097/01.PCC.0000149131.72248.E6

[13] Proulx, F., Joyal, J.S., Mariscalco, M.M., Leteurtre, S., Leclerc, F. and Lacroix, J. (2009) The Pediatric Multiple Organ Dysfunction Syndrome. Pediatr Crit Care Med, 10, 12-22. https://doi.org/10.1097/PCC.0b013e31819370a9

[14] Proulx, F., Fayon, M., Farrell, C.A., Lacroix, J. and Gauthier, M. (1996) Epidemiology of Sepsis and Multiple Organ Dysfunction Syndrome in Children. Chest, 109, 1033-1037. https://doi.org/10.1378/chest.109.4.1033

[15] Comstedt, P., Storgaard, M. and Lassen, A.T. (2009) The Systemic Inflammatory Response Syndrome (SIRS) in Acutely Hospitalised Medical Patients: A Cohort Study. Scand J Trauma Resusc Emerg Med, 17, 67. https://doi.org/10.1186/1757-7241-17-67

[16] Talmor, M., Hydo, L. and Barie, P.S. (1999) Relationship of Systemic Inflammatory Response Syndrome to Organ Dysfunction, Length of Stay, and Mortality in Critical Surgical Illness: Effect of Intensive Care Unit Resuscitation. Arch Surg, 134, 81-87. https://doi.org/10.1001/archsurg.134.1.81

[17] Wolfler, A., Silvani, P., Musicco, M., Antonelli, M. and Salvo, I. (2008) Incidence of and Mortality Due to Sepsis, Severe Sepsis and Septic Shock in Italian Pediatric Intensive Care Units: A Prospective National Survey. Intensive Care Med, 34, 1690-1697. https://doi.org/10.1007/s00134-008-1148-y

[18] Carvalho, P.R., Feldens, L., Seitz, E.E., Rocha, T.S., Soledade, M.A. and Trotta, E.A. (2005) Prevalence of Systemic Inflammatory Syndromes at a Tertiary Pediatric Intensive Care Unit. J Pediatr (Rio ), 81, 143-148. https://doi.org/10.2223/1320

[19] Watson, R.S., Carcillo, J.A., Linde-Zwirble, W.T., Clermont, G., Lidicker, J. and Angus, D.C. (2003) The Epidemiology of Severe Sepsis in Children in the United States. Am J Respir Crit Care Med, 167, 695-701. https://doi.org/10.1164/rccm.200207-682OC

[20] Dulhunty, J.M., Lipman, J. and Finfer, S. (2008) Does Severe Non-Infectious SIRS Differ from Severe Sepsis? Results from a Multi-Centre Australian and New Zealand Intensive Care Unit Study. Intensive Care Med, 34, 1654-1661.

https://doi.org/10.1007/s00134-008-1160-2

[21] Watson, R.S. and Carcillo, J.A. (2005) Scope and Epidemiology of Pediatric Sepsis. Pediatr Crit Care Med, 6, S3-5. https://doi.org/10.1097/01.PCC.0000161289.22464.C3

[22] Kim, J.E., Oh, S.H., Kim, K.M., Choi, B.H., Kim, D.Y., Cho, H.R., et al. (2010) Infections after Living Donor Liver Transplantation in Children. J Korean Med Sci, 25, 527-531. https://doi.org/10.3346/jkms.2010.25.4.527

[23] Hellinger, W.C., Crook, J.E., Heckman, M.G., Diehl, N.N., Shalev, J.A., Zubair, A.C., et al. (2009) Surgical Site Infection after Liver Transplantation: Risk Factors 
and Association with Graft Loss or Death.Transplantation, 87, 1387-1393. https://doi.org/10.1097/TP.0b013e3181a25133

[24] Lan, X., Zhang, H., Li, H.-Y., Chen, K.-F., Liu, F., Wei, Y.-G., et al. (2018) Feasibility of Using Marginal Liver Grafts in Living Donor Liver Transplantation. World J Gastroenterol, 24, 2551-2456. https://doi.org/10.3748/wjg.v24.i23.2441

[25] Miller, C.M., Quintini, C., Dhawan, A., Durand, F., Heimbach, J.K., Kim-Schluger, H.L., et al. (2017) The International Liver Transplantation Society Living Donor Liver Transplant Recipient Guideline. Transplantation, 101, 938-944. https://doi.org/10.1097/TP.0000000000001571

[26] Spapen, H. (2008) Liver Perfusion in Sepsis, Septic Shock, and Multiorgan Failure. Anat Rec(Hoboken), 291, 714-720. https://doi.org/10.1002/ar.20646

[27] Nguyen, H. and Hughes, C.B. (2009) Surgical Site Infection after Liver Transplantation: Risk Factors and Association with Graft Loss or Death. Transplantation, 87, 1387-1393. https://doi.org/10.1097/TP.0b013e3181a25133

[28] Cakir, M., Arikan, C., Akman, S.A., Baran, M., Saz, U.E., Yagci, R.V., et al. (2010) Infectious Complications in Pediatric Liver Transplantation Candidates. PediatrTransplant, 14, 82-86. https://doi.org/10.1111/j.1399-3046.2009.01136.x

[29] Tenza, E., Bernardo, C., Escudero, D., Otero, J., Quindós, B., Miyar, A., et al. (2009) Liver Transplantation Complications in the Intensive Care Unit and at 6 Months. Transplantation Proceedings, 41, 1050-1053. https://doi.org/10.1016/j.transproceed.2009.02.014

[30] Pavare, J., Grope, I. and Gardovska, D. (2009) Prevalence of Systemic Inflammatory Response Syndrome (SIRS) in Hospitalized Children: A Point Prevalence Study. BMC Pediatr, 9, 25. https://doi.org/10.1186/1471-2431-9-25

[31] Czubkowski, P., Socha, P. and Pawlowska, J. (2010) Current Status of Oxidative Stress in Pediatric Liver Transplantation. Pediatric Transplantation, 14, 169-177. https://doi.org/10.1111/j.1399-3046.2009.01256.x

[32] Dunne, J.R., Malone, D.L., Tracy, J.K. and Napolitano, L.M. (2004) Allogenic Blood Transfusion in the First 24 Hours after Trauma Is Associated with Increased Systemic Inflammatory Response Syndrome (SIRS) and Death. Surg Infect (Larchmt), 5, 395-404. https://doi.org/10.1089/sur.2004.5.395

[33] Pareja, E., Cortes, M., Navarro, R., Sanjuan, F., López, R. and Mir, J. (2010) Vascular Complications after Orthotopic Liver Transplantation: Hepatic Artery Thrombosis. Transplant Proc., 42, 2970-2972. https://doi.org/10.1016/j.transproceed.2010.07.063

[34] Ziaziaris, W.A., Darani, A., Holland, J.A., Angus, A.A., Karpelowsky, J., Shun, A. and Thomas, G. (2015) Delayed Primary Closure and the Incidence of Surgical Complications in Pediatric Liver Transplant Recipients. Journal of Pediatric Sur gery, 50, 2137-2140. https://doi.org/10.1016/j.jpedsurg.2015.08.045

[35] Yerdel, M.A., Gunson, B., Mirza, D., Karayalçin, K., Olliff, S., Buckels, J., Mayer, D, McMaster, P. and Pirenne, J. (2998) Portal Vein Thrombosis in Adults Undergoing Liver Transplantation: Risk Factors, Screening, Management, and Outcome. Transplantation, 69, 1873-1881. https://doi.org/10.1097/00007890-200005150-00023

[36] Cresswell, A.B., Jassem, W., Srinivasan, P., Prachalias, A.A., Sizer, E., Burnal, W., et al. (2012) The Effect of Body Position on Compartmental Intra-Abdominal Pressure Following Liver Transplantation. Annals of Intensive, 2, 12. https://doi.org/10.1186/2110-5820-2-S1-S12

[37] Cresswell, A.B. and Wendon, J.A. (2007) Hepatic Function and Non-Invasive Hepatosplanchnic Monitoring in Patients with Abdominal Hypertension. Acta Clin- 
Belg, Suppl 1, 113-118. https://doi.org/10.1179/acb.2007.62.s1.014

[38] Kaussen, T., Srinivasan, P.K., Afify, M., Herweg, C., Tolba, R., Conze, J. and Schachtrupp, A. (2012) Influence of Two Different Levels of Intra-Abdominal Hypertension on Bacterial Translocation in a Porcine Model. Ann Intensive Care, 2 , 17. https://doi.org/10.1186/2110-5820-2-S1-S17 